\title{
Estimation of losses caused by rice leaf folder, Cnaphalocrosis medinalis Guen. (Lepidoptera: Pyralidae) on paddy crop at Jagdalpur (Chhattisgarh)
}

\author{
CHANDRA SHEKHAR NETAM* AND A. K. GUPTA
}

Department of Entomology, College of Agriculture, Indira Gandhi Agricultural University, RAIPUR (C.G.) INDIA

\section{ARITCLE INFO}

Received : 11.07 .2017

Accepted : 30.09 .2017

\section{KEY WORDS :}

Estimation, Leaf folder, Paddy crop, Yield loss

*Corresponding author: Email : csnetam169@gmail.com

\section{ABSTRACT :}

Studies on estimation of losses caused by rice leaf folder on variety swarna presented regression analysis revealed a non significant negative relation between damaged flag leaves and grain yield in natural infestation $\left(r^{2}=0.186\right)$ while, a significant negative relation observed in augmented condition $\left(r^{2}=0.739\right)$.

How to view point the article : Netam, Chandra Shekhar and Gupta, A.K. (2017). Estimation of losses caused by rice leaf folder, Cnaphalocrosis medinalis Guen. (Lepidoptera: Pyralidae) on paddy crop at Jagdalpur (Chhattisgarh). Internat. J. Plant Protec., 10(2) : 461-464, DOI : 10.15740/ HAS/IJPP/10.2/461-464. 